\title{
Intermittent and Transient Hypotension-related Anterior Cord Syndrome following Elective Cervical Spine Surgery: A Case Report
}

\author{
Danielle Greig ${ }^{1}$, Stephen Zoller ${ }^{1}$, William L. Sheppard ${ }^{1}$, Don Young Park ${ }^{1}$
}

Learning Point of the Article:

Acute postoperative anterior cord syndrome can occur in extremely rare cases and vigilant neurological monitoring with close blood pressure monitoring and maintenance may improve the condition.

\section{Abstract}

Introduction: Anterior cord syndrome (ACS) is a type of incomplete spinal cord injury caused by either direct compression of the anterior spinal cord, or by ischemia of the anterior spinal artery. This phenomenon has neither been described transiently nor intraoperatively.

Case Report: We describe the case of a 61-year-old male who developed intermittent and transient anterior spinal cord syndrome secondary to hypotension related hypoperfusion of the anterior spinal artery after elective cervical spine surgery. Through close blood pressure monitoring and intensive care unit support, the patient regained full neurological recovery.

Conclusion: Anterior spinal cord syndrome is a rare condition affecting the anterior $2 / 3$ of the spinal cord, resulting in incomplete paralysis. Blood flow can be disrupted through the anterior spinal artery, either through thrombosis or hypotension. We describe our rare case so that surgeons may recognize this potentially devastating condition.

Keywords: Spine, hypoperfusion, hypotension, cord injury, anterior cord syndrome.

\section{Introduction}

Anterior cord syndrome (ACS) is a type of incomplete spinal cord injury in which the anterior two-thirds of the spinal cord is affected. It can be caused by direct compression of the anterior spinal cord, or by ischemia of the anterior spinal artery. Recovery is unpredictable and management is largely supportive $[1,2]$.

ACS has also been noted to occur intraoperatively. Neuromonitoring changes consistent with deficits specific to ACS have been reported during corrective deformity surgery for scoliosis and kyphosis [3]. This phenomenon has been attributed to iatrogenic injury. ACS specifically has been attributed to perfusion-based deficits, and often manifest with decreases in bilateral motor potentials with preserved sensory potentials on neuromonitoring $[3,4,5]$.

We describe the case of a 61-year-old male who underwent elective cervical surgery and suddenly developed neurologic deficits consistent with ACS. This case is unique in that the patient's neurologic deficits appeared to wax and wane in response to changes in his blood pressure (BP) in the immediate post-operative course. This case report highlights the importance of close clinical monitoring postoperatively in cervical spine surgery, especially in at-risk patients. The purpose of this case report is to detail findings of acute postoperative ACS, which has not been well described in the literature to-date.

\section{Case Report}

The patient was a 61-year-old Caucasian male with a history of coronary artery disease and hyperlipidemia, who initially presented with right shoulder and arm pain, as well as numbness and tingling. He was very active, working as a tennis instructor without any neurological deficits. On exam, motor/sensation was fully intact throughout both upper and lower extremities,
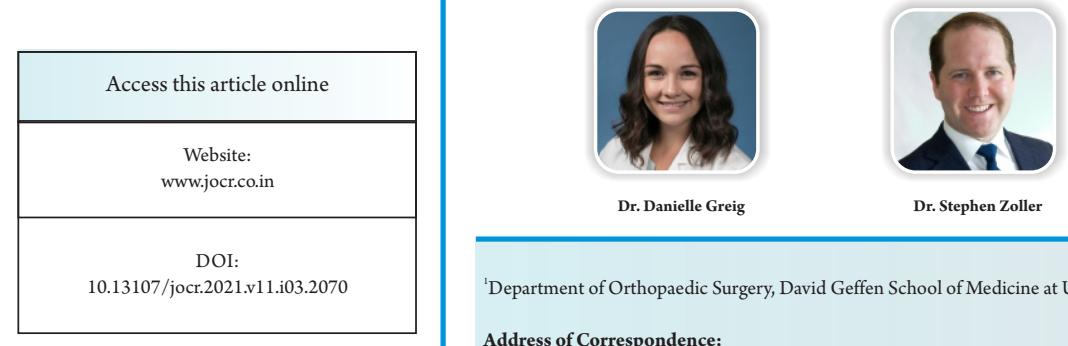

Dr. Stephen Zoller

Address of Correspondence:

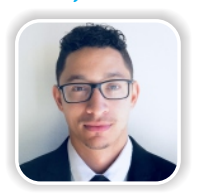

Dr. William L. Sheppard

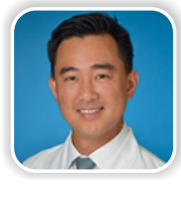

Dr. Don Young Park

'Department of Orthopaedic Surgery, David Geffen School of Medicine at UCLA, 1250 16th Street, Santa Monica, CA, 90404, USA.

Dr. Don Young Park,

Department of Orthopaedic Surgery, David Geffen School of Medicine at UCLA, 1250 16th Street, Santa Monica, CA, 90404, USA.

E-mail: DYPark@mednet.ucla.edu

Journal of Orthopaedic Case Reports | pISSN 2250-0685 | eISSN 2321-3817 | Available on www.jocr.co.in | doi:10.13107/jocr.2021.v11.i03.2030 This is an Open Access article distributed under the terms of the Creative Commons Attribution Non-Commercial License (http://creativecommons.org/licenses/by-nc/3.0) which permits unrestricted non-commercial use, distribution, and reproduction in any medium, provided the original work is properly cited. 


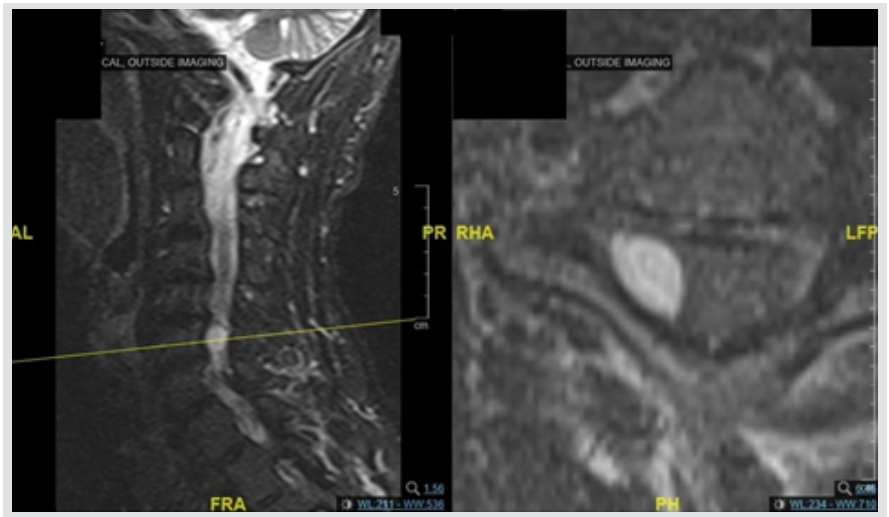

Figure 1: Pre-operative T2-weighted sagittal (a) and axial (b) magnetic resonance imaging of the cervical spine demonstrating a cystic mass occupying the right side of the spinal canal posterior to the C6 vertebral body. The cystic mass is causing significant compression of the spinal cord and the exiting nerve root on the right side.

with no upper motor neuron signs. He was classified as American Spinal Injury Association class E, Nurik Grade 0, with a full mJOA score. Despite this, the patient had failed conservative measures such as over-the-counter antiinflammatory medications, but had not received prior spinal injection nor surgical intervention. Advanced imaging was obtained. Magnetic resonance imaging (MRI) was notable for a cystic mass within the spinal canal on the right side posterior to the C6 vertebral body, causing spinal cord compression and right neural foraminal stenosis (Fig. 1a, b). This was attributed to synovial facet cyst formation. Given failed conservative measures, patient preference, and clear evidence on imaging, surgical intervention was recommended. The surgical plan was for C6 laminectomy decompression, right-sided C6-7 foraminotomy, and surgical excision and biopsy of mass under general anesthesia with intraoperative neuromonitoring.

The C6-7 laminectomy and foraminotomy were performed and the cystic mass was visualized on the right side, compressing the dura. The mass was isolated and excised. Pathology revealed a synovial cyst. Motor and sensory evoked potentials remained at baseline with no changes throughout the entire procedure. Estimated blood loss was $100 \mathrm{cc}$. A subfascial drain was also placed which was removed on POD2. Output was $275 \mathrm{cc}$ in the first $24 \mathrm{~h}$, and then slowed to $40 \mathrm{cc}$ over the second $24 \mathrm{~h}$. Approximately $45 \mathrm{~min}$ after the procedure, he had full strength and sensation in the bilateral upper and lower extremities.

Two hours postoperatively; the patient complained that he was unable to move his arms or his legs after being sat up by nursing staff. He was found to have $0 / 5$ strength throughout all motor groups in the bilateral lower extremities, dullness to sensation throughout the bilateral lower extremities, $5 / 5$ deltoid strength bilaterally, $4 / 5$ biceps strength bilaterally, and $3 / 5$ triceps, wrist flexor/extensor, and interossei strength bilaterally. Patellar and Achilles reflexes were absent. The mean arterial pressure (MAP) before this episode was 91. Leading diagnosis at this time was ACS due to transiently decreased perfusion related to

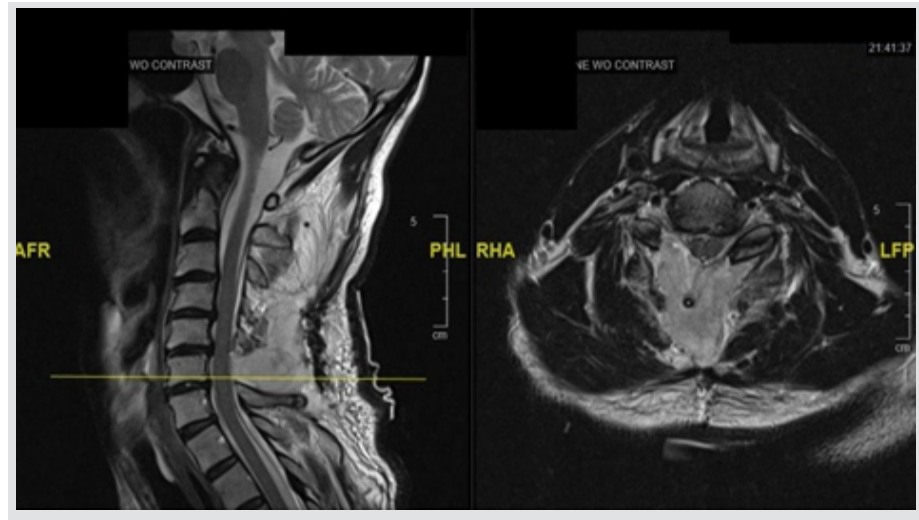

Figure 2: Post-operative T2-weighted sagittal (a) and axial (b) magnetic resonance imaging (MRI) of the cervical spine demonstrating post-operative changes with complete resection of the cystic mass and improvement of the cervical stenosis seen in the pre-operative MRI. There is no evidence of spinal cord compression or myelomalacia seen.

hypotension.

Ten minutes following onset of his weakness, he was found to have near full strength and sensation, with the exception of $4 / 5$ triceps, wrist extensors, wrist flexors, and interossei. The MAP, at this time, was 101. After another $10 \mathrm{~min}$, he again had recurrence of the neurological deficits. The MAP was measured at 93. This second episode resolved in approximately 10 additional min. BP readings were obtained per Recovery Unit protocol. In the Recovery Unit, these measures are obtained every 5-10 min. An urgent MRI was performed, which demonstrated expected post-surgical changes without evidence of cord signal change or compression and no compressive hematoma. In addition, there was a low likelihood of postoperative hematoma given the transient/intermittent symptoms described above (Fig. 2a, b).

The patient was admitted to the intensive care unit (ICU) for close monitoring of both his BP ( 2 min cycles) and neurologic exam (whenever MAP $<95$, otherwise every $1-2 \mathrm{~h}$ per ICU protocol). He was given one dose of steroids postoperatively (dexamethasone $10 \mathrm{mg} \mathrm{IV}$ ) in response to his symptoms as reperfusion injury was suspected. MAPs were maintained in the high $90 \mathrm{~s}$ without the need for pressure support (norepinephrine or alternative therapies). High dose steroid treatment was not utilized. Over the next $24 \mathrm{~h}$, his neurologic exam stabilized at full strength and sensation, with no recurrence of neurologic deficits. He had complete resolution of his pre-operative symptoms with normal neurological function and returned to teaching tennis over 1 year after surgery.

\section{Discussion}

ACS is a rare but devastating spinal cord injury that can be caused by occlusion or hypoperfusion of the anterior spinal artery. The anterior spinal artery is comprised anastomoses between ascending and descending branches of large anterior radicular arteries $[4,6]$. It gives off anterior sulcal arteries within the spinal cord, which supply all of the spinal cord gray matter 
except the posterior horns, as well as the anterior and lateral white matter columns $[6,7,8]$. There is no significant collateral blood flow between the anterior and posterior circulations $[4,6$, 7].

Clinically, ACS presents as the abrupt onset of flaccid paralysis, usually affecting the lower extremities more than the upper extremities, areflexia, and loss of pain and temperature sensation. Sparing of the posterior columns, which are vascularized by the posterior circulation, results in preservation of proprioception and vibratory sensation. The most common etiology is thought to be arteriosclerosis, with less prevalent causes including aortic pathology, disc herniation, acute systemic hypotension, spinal surgery, and vascular procedures $[5,6,9]$. Barrera et al. found that $36.1 \%$ of cases were idiopathic, with no identifiable vascular risk factors. It is more common at thoracic levels (55\%) and is rare in the cervical spine (2.7\%) [5]. Treatment is directed at the underlying cause, but for the most part is supportive with induced hypertension to maximize cord perfusion with vasopressors $[1,2]$.

Prognosis of an ACS is generally very poor. Foo et al. found that a mean of $60 \%$ of patients regain some motor function, but complete motor return is unusual [10]. Patients with some preservation of motor function below the level of the lesion have a favorable prognosis when compared with those with complete motor loss. At a mean follow-up of 3 years, Robertson et al. found that $42 \%$ of patients required a wheelchair, $54 \%$ required catheterization, and $29 \%$ had pain [11]. Mortality is as high as $23 \%$ and increased in patients with severe neurologic impairment at presentation.

We hypothesize that our patient's presentation is secondary to relative hypotension causing decreased perfusion to the anterior spinal cord postoperatively. Sudden hypotension might have caused reflex vasoconstriction to elevate the BP, which could have resulted in occlusion of the anterior spinal artery, resulting in sudden onset weakness. The change was reversible, as elevation of $\mathrm{BP}$ might have re-opened the anterior spinal artery, resulting in neurological recovery due to perfusion of the cord. Often, hematoma can present in a similar presentation. However, given no evidence on MRI and drainage tube placement with adequate output, hematoma is unlikely. The patients symptoms started when he was seated in the postanesthesia care unit (from supine to sitting position), making compression less likely as this position change would have the opposite effect (less compression sitting than supine). Literature review revealed only one published case of hypotension-related ACS. Schneider [12] describes the case of a 48-year-old male who presented to the emergency department (ED) with neck pain and lower extremity weakness $2 \mathrm{~h}$ after given a dose of atenolol for hypertension. His BP was initially 190/110, and at the time of his arrival at the ED was found to be 110/60 with the neurologic exam consistent with ACS. The MRI demonstrated a disc herniation at C6-7 causing severe spinal canal stenosis. The author hypothesizes that ACS was caused by cervical stenosis and relative hypotension after treatment with atenolol, which did not improve despite aggressive MAP support.

Our patient's neurologic deficits developed after sitting up, which may have induced orthostatic hypotension. His MAPs were in the low 90s during the episodes of deficit, which then improved when his MAPs were in the low 100s. At his three most recent pre-operative visits before surgery, his MAP baseline was in the high 90s to low 100s. For this patient, a MAP of low 90s may be relatively hypotensive. The patient had a known history of coronary artery disease and hyperlipidemia, which may pre-dispose him to arterial insufficiency. Surgical intervention may have unmasked the patient's arterial insufficiency and left him unable to compensate appropriately with the relative hypotension. Clinicians should maintain a low index of suspicion for suspecting ACS in patients with vascular disease, and screening for cervical vascular disease may be warranted in the appropriate patient population.

An additional potential diagnosis that may explain the patient's condition is spinal cord reperfusion injury, which is due to reperfusion of a chronically ischemic spinal cord from longstanding spinal cord compression. This clinical entity can rarely occur after spinal cord decompression without direct cord injury, with several case reports leading to paraplegia and even quadriplegia [13]. These cases reported incomplete spinal cord injuries that partially improved over time with high-dose steroid treatment and rehabilitation. The condition is also known as "white cord syndrome" due to the hyperintensity seen within the spinal cord on T2-weighted MRI sequences after cervical decompression. In our case, however, there was no evidence of hyperintensity in the pre-operative or postoperative MRI studies and the clinical picture was more consistent with ACS.

Several publications have proposed clinical checklists to improve clinical outcome in the event of spinal cord injury. The use of a hemodynamic safety checklist to maintain MAP goals of $85-90 \mathrm{~mm} \mathrm{Hg}$ within the first 7 days after SCI demonstrated improved hemodynamic management of spinal cord injured patients [14]. Guidelines provided by the Consortium for Spinal Cord Medicine further recommends immediate management of systolic BP (SBP) <90, fluid resuscitation to balance base deficit and elevated lactate levels, and the use of vasopressors if fluid response is inadequate to maintain SBP $>90$ [15]. Other measures include treating symptomatic bradycardia, prevent hyperthermia, frequent examinations, and repeat imaging should significant changes occur. High-dose steroids are not part of the guidelines and are not utilized in this 
institution, given recent data demonstrating increased risk of gastrointestinal hemorrhage, acute respiratory distress syndrome, and various respiratory tract infections [16].

A limitation of our case is lack of direct evidence of a hypoperfusion-related ACS, such as an MR angiogram or MRI diffusion-weighted imaging. Conventional MRI sequences will reveal ischemic lesions only after $10-15 \mathrm{~h}$ from symptom onset. However, diffusion-weight imaging is effective in diagnosing spinal cord ischemia within minutes of symptom onset. Furthermore, we did not have arterial BP monitoring in this case, which could provide real-time documentation of the MAPs.
Conclusion

We describe an unusual and rare case of an intermittent and transient ACS related to relative hypotension following cervical spine surgery. Our case is the only case in the literature describing post-operative ACS due to hypotension with full resolution of neurological deficits.

\section{Clinical Message}

Close monitoring of $\mathrm{BP}$, with permissive hypertension is recommended to avoid this potentially devastating complication.

\section{References}

1. Rose JC, Stephan AM. Optimizing blood pressure in neurological emergencies. Neurocritical Care 2004;1:287300 .

2. Teitelbaum JS. Management of blood pressure in acute neurological illnesses. In: Noseworthy JH, editor. Neurological Therapeutics: Principles and Practice. London, UK: Martin Dunitz; 2003.p. 1148-60.

3. Lewis SJ, Wong IH, Strantzas S, Holmes LM, Vreugdenhil I, Bensky $\mathrm{H}$, et al. Responding to intraoperative neuromonitoring changes during pediatric coronal spinal deformity surgery. Global Spine J 2019;9:15S-21.

4. Lazorthes G, Gouaze A, Zadeh JO, Santini JJ, Lazorthes Y, Burdin P. Arterial vascularization of the spinal cord. Recent studies of the anastomotic substitution pathways. J Neurosurg 1971;35:253-62.

5. De La Barrera SS, Barca-Buyo A, Montoto-Marqués A, Ferreiro-Velasco ME, Cidoncha-Dans M, RodriguezSotillo A. Spinal cord infarction: Prognosis and recovery in a series of 36 patients. Spinal Cord 2001;39:520-5.

6. Sandson TA, Joseph HF. Spinal cord infarction. Medicine 1989;68:282-92.

7. Gillilan LA. The arterial blood supply of the human spinal cord.J Comp Neurol 1958;110:75-103.

8. Herren RY. Sulcal and intrinsic blood vessels of human spinal cord. Arch Neurol Psychiatry 1939;41:678.
9. Cheshire WP, Santos CC, Massey EW, Howard JF Jr. Spinal cord infarction: Etiology and outcome. Neurology 1996;47:321-30.

10. Foo D, Alain BR. Anterior spinal artery syndrome and its natural history. Spinal Cord 1983;21:1-10.

11. Robertson CE, Brown RD Jr., Wijdicks EF, Rabinstein AA. Recovery after spinal cord infarcts: Long-term outcome in 115 patients. Neurology 2011;78:114-21.

12. Schneider GS. Anterior spinal cord syndrome after initiation of treatment with atenolol. J Emerg Med 2010;38:e49-52.

13. Chin KR, Seale J, Cumming V. White cord syndrome of acute tetraplegia after anterior cervical decompression and fusion for chronic spinal cord compression: A case report. Case Rep Orthop 2013;2013:697918.

14. Sewell MD, Vachhani K, Hockings J, Chan J, Alrawi A, Williams R. A hemodynamic safety checklist can improve blood pressure monitoring in patients with acute spinal cord injury. World Neurosurg 2019; 128:225-30.

15. Consortium for Spinal Cord Medicine. Early acute management in adults with spinal cord injury: A clinical practice guideline for health-care professionals. J Spinal Cord Med 2008;31:403-79.

16. Liu Z. High-dose methylprednisolone for acute traumatic spinal cord injury: A meta-analysis. Neurology 2019;93:e841-50.

Conflict of Interest: Nil

Source of Support: Nil

Consent: The authors confirm that informed consent was obtained from the patient for publication of this case report

\section{How to Cite this Article}

Greig D, Zoller S, Sheppard WL, Park DY. Intermittent and Transient Hypotension-related Anterior Cord Syndrome following Elective Cervical Spine Surgery: A Case Report. Journal of Orthopaedic Case Reports 2021 March;11(3):21-24 